\title{
Neutron performance analysis for ESS target proposal
}

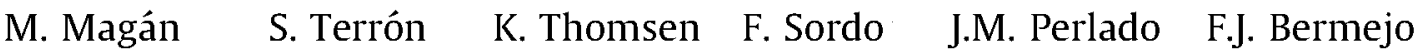

\begin{abstract}
A B S T R A C T
In the course of discussing different target types for their suitability in the European Spallation Source (ESS) one main focus was on neutronics' performance. Diverse concepts have been assessed baselining some preliminary engineering and geometrical details and including some optimization. With the restrictions and resulting uncertainty imposed by the lack of detailed designs optimizations at the time of compiling this paper, the conclusion drawn is basically that there is a little difference in the neutronic yield of the investigated targets. Other criteria like safety, environmental compatibility, reliability and cost will thus dominate the choice of an ESS target.
\end{abstract}

\section{Introduction}

Wide spread investigations have taken place starting with the ESS Preparatory Phase Study aimed at selecting the best suitable target concept for the European Spallation Source (ESS). One focus of this process, evidently, lies on the expected neutronic yield under the specific ESS conditions. Diverse numerical models have been compiled for a wide range of different possible target concepts. Facing the lack of engineering details, material choices and geometries have been based on principal requirements, e.g., concerning the suitability of particular materials, and on rough estimates for dimensions stemming from cooling requirements. Although at the current state these boundary conditions are not known at much detail, first simulations can give a clear indication of significant differences between the performances of the diverse approaches and, in case, can rule out certain options. In order to enhance the validity of the reported comparisons, some optimization has been performed individually for each concept, i.e., premoderator thickness, moderator dimensions, relative position between moderator and target and reflector dimensions have been varied to obtain near optimal performances. The obtained results allow for some meaningful benchmarking and at the same time give an indication of the margins for optimization of the different target types.

The following target variants have been investigated:

- Liquid metal (mercury, lead eutectics).

- Solid rotating target with cold plates (water cooled).

- Solid rotating target cooled by helium.

- Cannelloni target.

All calculations have been performed assuming the ESS beam parameters as available in 2010: Gaussian profile with 2 . $\sigma_{x}=10,2 \cdot \sigma_{y}=3$ (in $\mathrm{cm}$ ), $2.5 \mathrm{GeV}$ per proton, and $5 \mathrm{MW}$ beam power. The accelerator fires at a rate of $20 \mathrm{~Hz}$, making the total energy per pulse $250 \mathrm{~kJ}$. In addition to the expected long pulses (1 ms duration) the response for short pulses has been simulated too, thus obtaining more information on the fine-scale timing.

\section{Methodology}

In order to analyze the neutronics of the target-moderatorreflector assembly, several MCNPX models [1] have been developed based on SNS-STS proposal [2]. This configuration presents a Coupled Wing moderator with the following main parameters: three lines with $120 \mathrm{~cm}^{2}$ of moderator surface view, a cylinder of pure parahydrogen at $22 \mathrm{~K}$ as moderator, light water as premoderator, beryllium cooled by heavy water ( $5 \%$ in volume) as reflector and several $\mathrm{Al}_{3} \mathrm{Mg}$ claddings. Moderator height has been set to the view height, as increasing it reduces neutron performance. 
This geometry will be similar to the ESS final geometry, since parahydrogen moderators maximize the neutron flux in the range of interest [3] and their performance increases when a water premoderator is included [4]. Concerning the configuration of the moderator, Wing configuration reduces high energy neutrons background.

Fig. 1 shows the geometry used for the moderator-reflector assembly simulations.

The latest edition of the Los Alamos National Laboratory scattering kernel is applied [5] together with the ENDEF-VII cross-sections libraries [6]. There are several isotopes in which proton cross-sections are not included in this data library, in these cases TENDEL-2010 [7] has been used. For high energy reactions (above $20 \mathrm{MeV}$ ), the intranuclear cascade model CEM [8] is applied.

The reference figure of merit studied for the optimization is the "Time integrated neutron flux below $5 \mathrm{meV}$ " on the moderator's surface. This figure has been used in other optimization studies $[2,9,10]$ and has been found to be a representative figure of the assembly performance. An optimization loop has been carried out for each target design considering the main geometrical parameters, i.e., relative position of target and moderator, moderator radius, premoderator thickness and reflector dimensions. Therefore, figures of neutron performance have been calculated close to the optimal configuration. Since dozens of simulations need to be done for each target type, we need a figure of merit that is computationally cheap to find the optimal configuration, and, then, we can do a fine energy binning in order to have a more detailed characterization of the brightness. Concerning premoderator, only the target-side thickness has been optimized because far-target-side $(5 \mathrm{~mm})$ and lateral-side $(10 \mathrm{~mm})$ effects will be much lower than the first one [11].

Time integrated neutron flux and neutron time distributions have both been evaluated by means of a point detector placed $10 \mathrm{~m}$ away from the moderator surface. The point detector was enclosed in a collimator, using cells with zero importance, to avoid indirect contributions. The collimator is sized so that all neutrons at the point detector need to come from the moderator surface. The time binning was influenced by a user supplied TALLYX subroutine such that the moderator emission time (time at which the neutrons exit the moderator) was scored rather than the arrival time at the detector point. This detector modification is known in literature under the name time-of-flight-corrected point detector $[12,13]$. Neutron time distribution is calculated for energies within 4.5 and $5.5 \mathrm{meV}$.

Optimization has been performed for each variable, as crosseffects have been shown to be small, so we can consider the brightness as a product of independent variables, with sufficient accuracy for our purposes. The entire array of results, resulting from the optimization of each parameter for each target, is too large for this paper to show, but Figs. 2 and 3 show the trend of most variables. The slopes around the maximum are not steep, meaning that, from an engineering point of view, it is possible to change the parameters around the optimum without a great sacrifice of neutron performance.

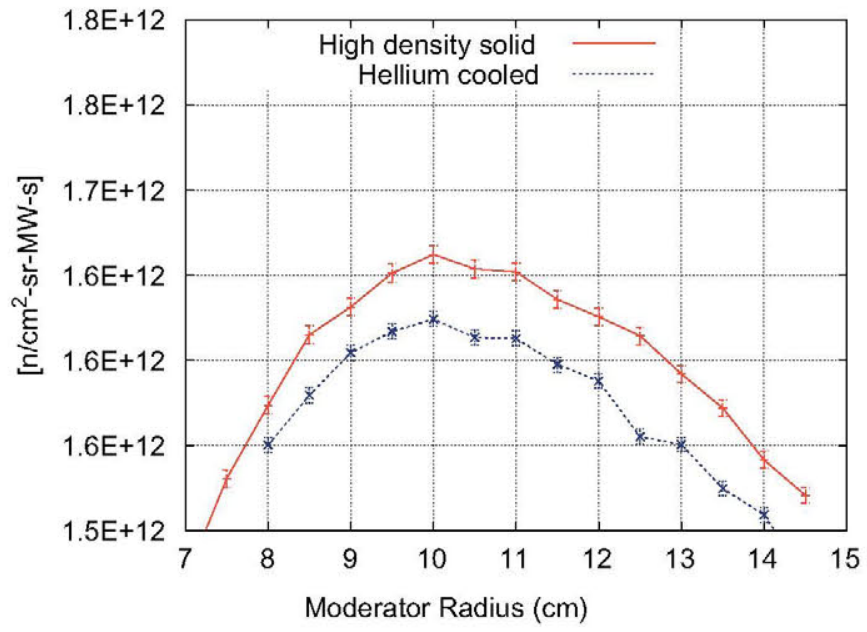

Fig. 2. Sensitivity of target performance to moderator radius.

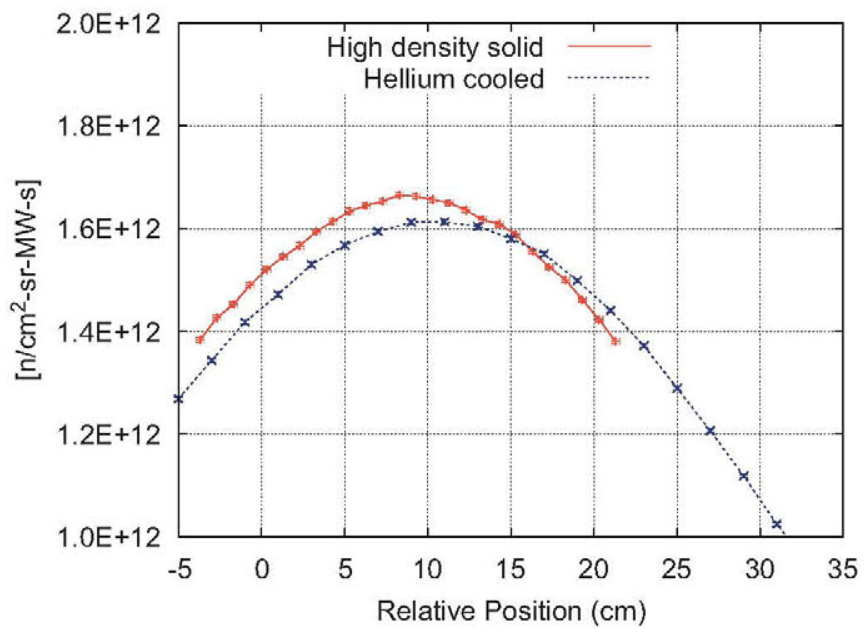

Fig. 3. Sensitivity of target performance to moderator position.
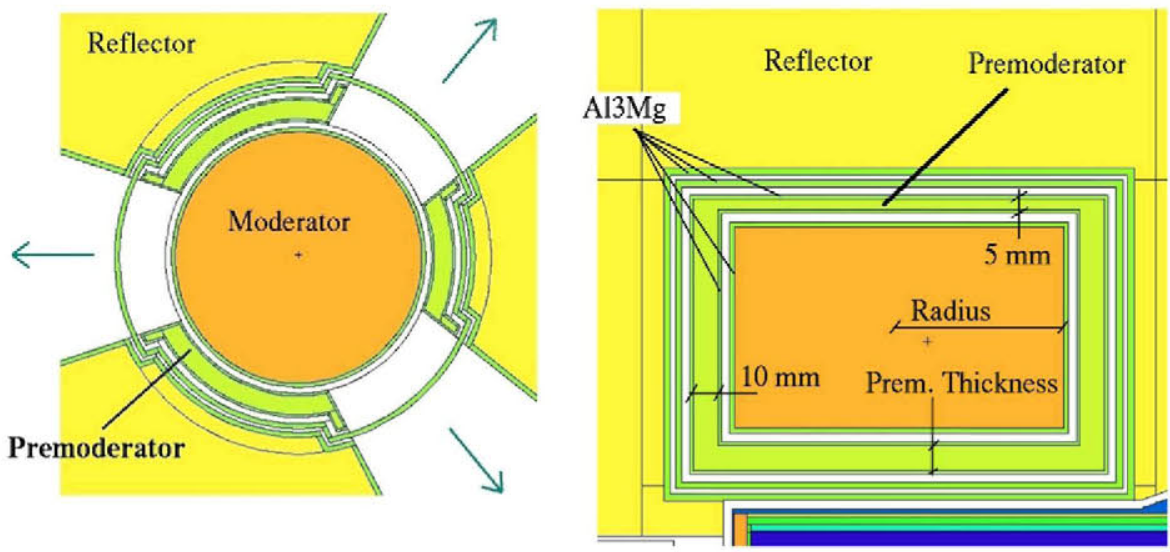

Fig. 1. Wing parahydrogen moderator geometry. 


\section{Liquid metal targets}

Liquid metal targets have been one of the most accepted options in high power neutron sources (JSNS [14] and SNS [15]) and this concept was the main design option for the European neutron source in 2003 [16]. The MCNPX model is based on ESS2003 design and it includes three layers of steel $(3 \mathrm{~mm}$ thick each), a helium gap $(2 \mathrm{~mm})$ and a light water channel $(3 \mathrm{~mm})$. This multiple barrier system is needed in order to avoid liquid metal spread in case of failure of the first enclosure. Fig. 4 shows the geometry analyzed for liquid targets.

Concerning target materials, the following candidates have been studied:

- Mercury: Proposed for ESS in 2003 and in operation in SNS and JSNS spallation sources.

- Lead and lead alloys: Interesting candidates for spallation targets, especially lead-bismuth, for which a large operational experience has been accumulated in fission reactors and during the MEGAPIE project [17].

It is possible to consider several other candidates but these are the most relevant ones, so analyzing them allows us to have an overview of the liquid metal target neutron efficiency.

For each target material an optimization loop has been performed in order to have a representative neutron performance value. Table 1 shows final values for the optimization.

Fig. 5 shows the time distribution of neutron brightness on the moderator surface for the optimized configuration of each option for instantaneous pulse and $1 \mathrm{~ms}$ pulse length. In both cases, short and long proton pulses, the differences are rather small, and unless the researchers are looking for the highest performance, they should not be the main criteria to decide the target material. Engineering and safety constraints should be considered first.

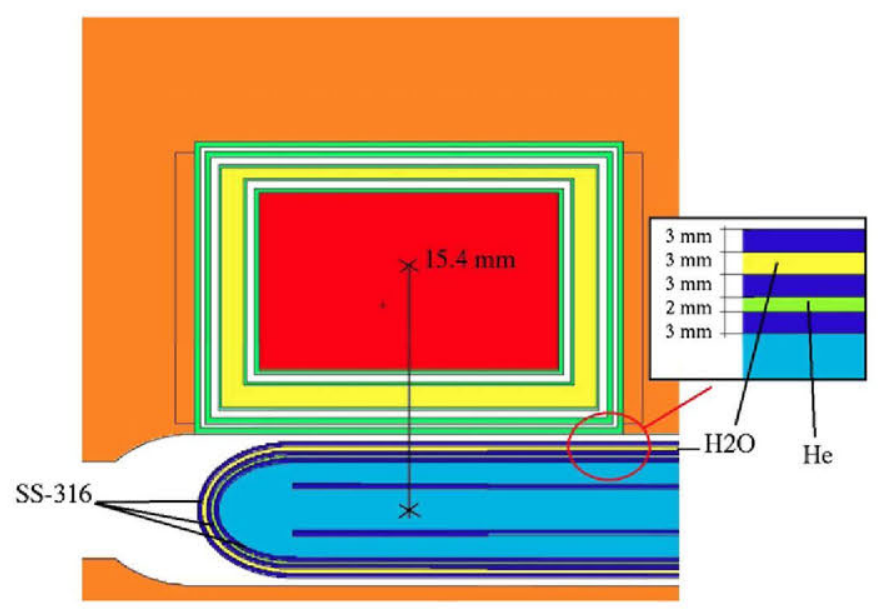

Fig. 4. Liquid target geometry.

\section{Solid rotating target cooled by cold plates}

The solid rotating target cooled by cold plates proposed by ESS-Bilbao [18] is an extension of the design proposed for SNSSTS [19]. From a neutronic point of view, the main advantage of this concept is that it allows to maximize the density of the target material. Higher density results in a brighter neutron source, increasing the neutron flux in the moderator. The MCNPX model analyzed in this document consists of several layers around a tungsten (Wolfmet HE397) nucleus. This geometry is intended to be a representative of the real configuration and allows us to ponder the neutronic effect of its different elements, i.e., two layers of $\mathrm{Al}_{3} \mathrm{Mg}$ cladding ( 2 and $5 \mathrm{~mm}$ thick each), homogenized cooling channels ( $4 \mathrm{~mm} \mathrm{Al}_{3} \mathrm{Mg}+$ Water) and SS-316 steel cladding ( $3 \mathrm{~mm}$ thick). Light water is used as a coolant, since it serves as a first layer of premoderator. While heavy water could be used instead, we would need to increase premoderator thickness in that case, leading to the same results.

Three compositions for target material have been studied:

- Tungsten with $95 \%$ of nominal density;

- Tungsten with $75 \%$ of nominal density;

- Tungsten homogenized with light water (75\% of tungsten in volume).

The 95\% density target corresponds to the cold plates design which seeks for the highest target density in order to maximize neutron performance. The $75 \%$ density option corresponds to gascooled rods (helium), and finally, the last proposal represents tungsten rods cooled by light water in a cross flow scheme. Fig. 6 shows MCNPX model for solid rotating targets.

For each of the cases analyzed, an optimization loop over the main design parameters has been carried out. The values of the design parameters collected in Table 2 represent good approximations to optimal configurations.

Fig. 7 shows the comparison between high density tungsten (95\%), low density tungsten (75\%) and low density tungsten with water ( $75 \%$ tungsten and $25 \%$ water) in terms of neutron brightness on the moderator surface, calculated with the design parameters in Table 2 . For the $75 \%$ density case, there is a significant reduction $(\sim 15 \%)$ in neutron performance. When light water is introduced in the target, there is a stronger decrease $(\sim 30 \%)$ in neutron performance due to thermal neutron captures.

\section{Solid rotating cooled by helium}

High velocity helium cooling is one of the options considered during the conceptual design phase of the ESS target. This option is expected to present some advantages in comparison to water cooling, since helium is chemically inert and would experience no phase transition in case of an accidental event. However, there are some remarkable challenges from an engineering point of view as well, e.g., high pumping power, high gas velocities in the coolant,

Table 1

Final parameters after optimization process for liquid metal targets.

\begin{tabular}{|c|c|c|c|c|}
\hline Parameter & Mercury & Lead & Lead-Bismuth & Lead-gold \\
\hline Moderator radius $(\mathrm{cm})$ & 10.0 & 10.0 & 10.0 & 10.0 \\
\hline Relative position $(\mathrm{cm})$ & 14.0 & 17.0 & 18.0 & 15.0 \\
\hline Premoderator thickness $(\mathrm{cm})$ & 1.5 & 1.0 & 0.75 & 1.25 \\
\hline Reflector radius $(\mathrm{cm})$ & 80 & 80 & 80 & 80 \\
\hline Reflector height (cm) & 80 & 80 & 80 & 80 \\
\hline Cold neutron ( $\mathrm{n} / \mathrm{cm}^{2} \mathrm{Sr} \mathrm{MW} \mathrm{s}$ ) & $1.80 \times 10^{12}$ & $1.78 \times 10^{12}$ & $1.77 \times 10^{12}$ & $1.63 \times 10^{12}$ \\
\hline
\end{tabular}




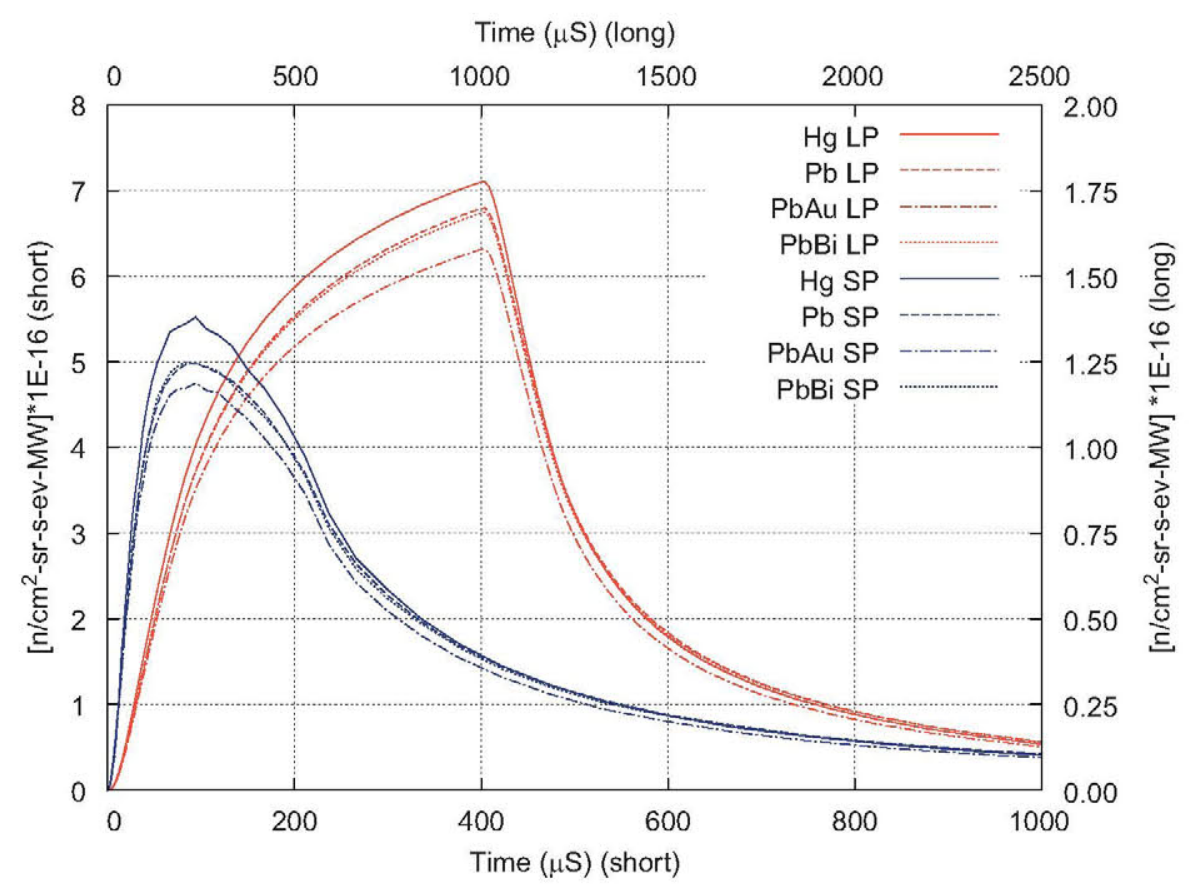

Fig. 5. Neutron pulse for liquid metals, for instantaneous and $1 \mathrm{~ms}$ pulse.

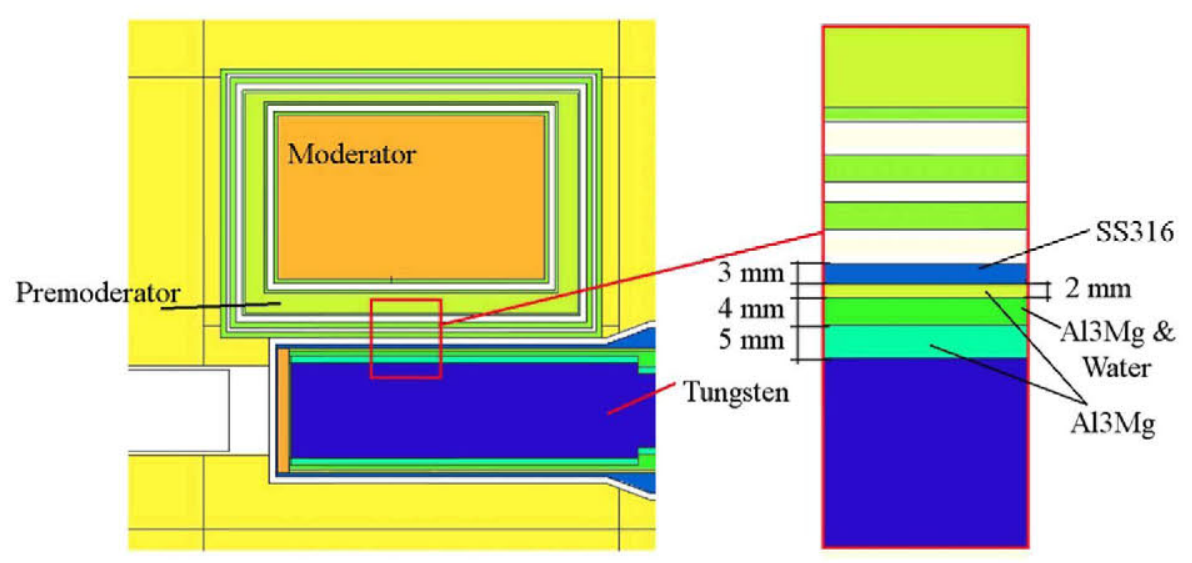

Fig. 6. Solid rotating model.

Table 2

Final parameters after optimization process for cold plate.

\begin{tabular}{llll}
\hline Parameter & High density & Low density & Low dens. water \\
\hline Moderator radius $(\mathrm{cm})$ & 10.0 & 10.0 & 10.5 \\
Relative position $(\mathrm{cm})$ & 9.0 & 10.0 & 9.0 \\
Premoderator thickness $(\mathrm{cm})$ & 1.5 & 1.5 & 1.0 \\
Reflector radius $(\mathrm{cm})$ & 70 & 70 & 70 \\
Reflector height $(\mathrm{cm})$ & 70 & 70 & 70 \\
Cold neutron $\left(\mathrm{n} / \mathrm{cm}^{2}\right.$ Sr MW s) & $1.78 \times 10^{12}$ & $1.53 \times 10^{12}$ & $1.25 \times 10^{12}$ \\
\hline
\end{tabular}

and lack of extensive operational experience in helium cooled spallation sources.

In order to analyze the neutronics of this kind of targets, the model shown in Fig. 8 has been developed. This model features two helium inlet channels (upper and lower sides) and one porous central area with the target material. In the absence of a more detailed description of the system, two different densities for the central area have been modeled:
- $90 \%$ of tungsten density, representing tungsten bricks with small helium channels between them, or a combination of cylinders of two different radii maximizing the packing;

- $75 \%$ of tungsten density, representing solid tungsten rods cooled externally.

Analogously to the precedent analyses, an optimization loop has been carried out for both density options. As shown in Table 3, moderator relative position is the only design parameter whose optimal value differs from one case to the other one. This effect is due to the fact that, when system density changes the neutron emission peak moves slightly away.

In Fig. 9 the effect of target density is noticeable, inducing around a $15 \%$ loss in terms of neutron brightness.

\section{Cannelloni target}

Cannelloni target is an evolution of the concept successfully used in SINQ [20]. Its design is based on lead rods inside zircalloy tubes 


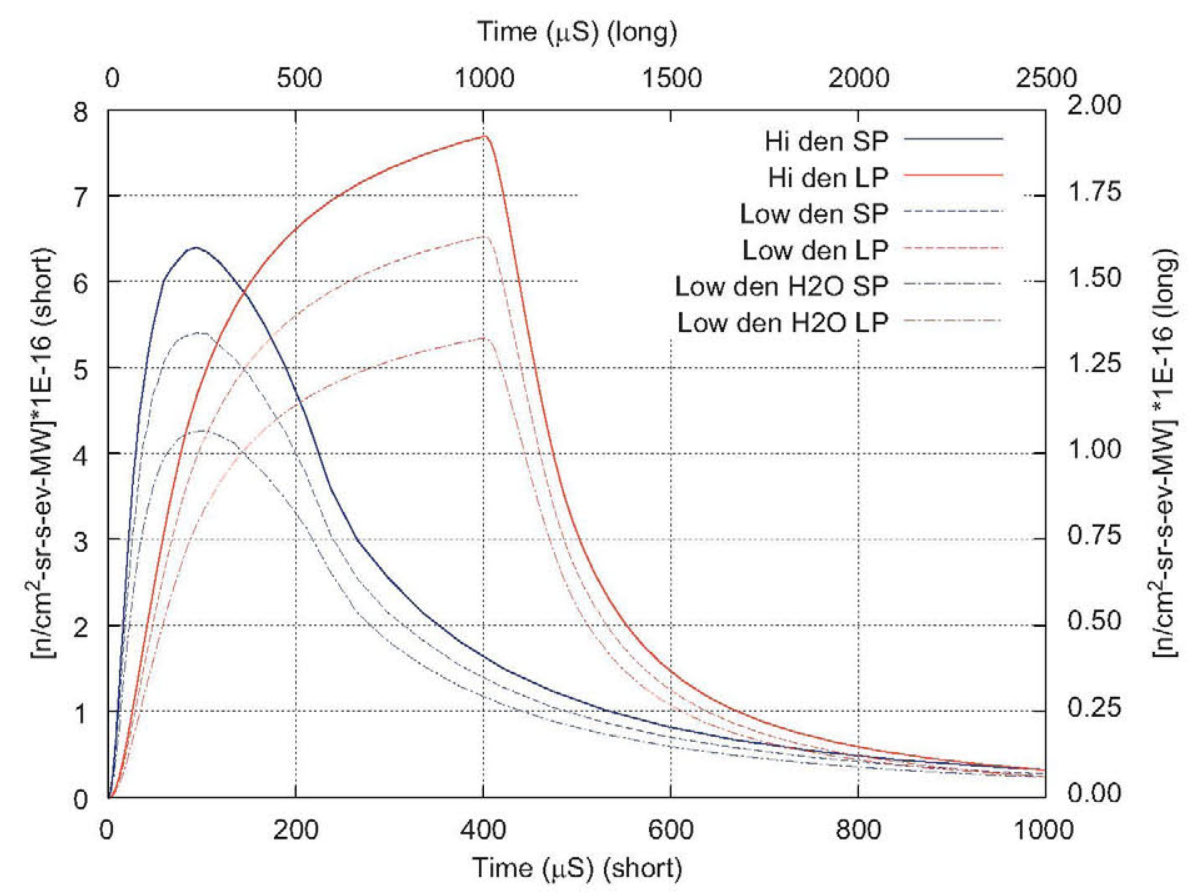

Fig. 7. Neutron pulse for solid targets, for instantaneous and $1 \mathrm{~ms}$ proton beam pulse.

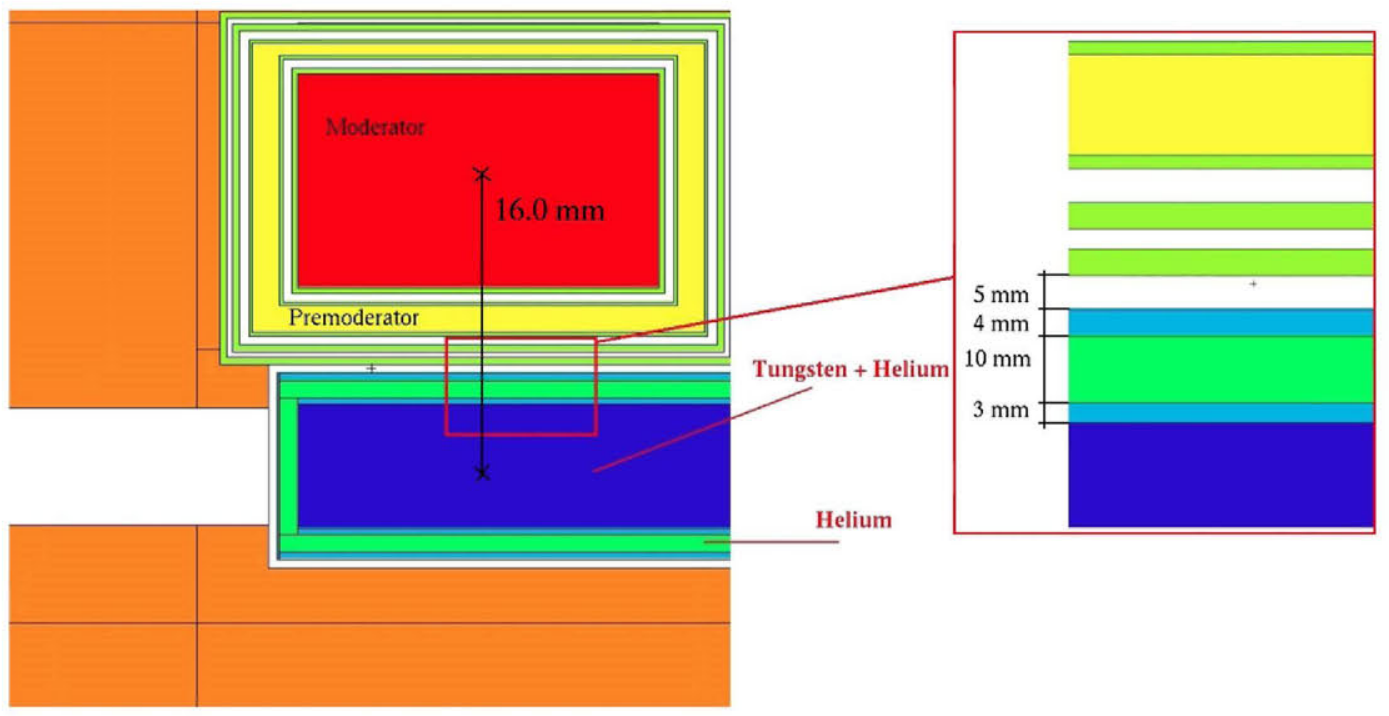

Fig. 8. Helium cooled target geometry.

Table 3

Final parameters after optimization process for helium cooled target.

\begin{tabular}{lll}
\hline Parameter & $90 \%$ density value & $75 \%$ density \\
\hline Moderator radius $(\mathrm{cm})$ & 10.0 & 10.0 \\
Relative position $(\mathrm{cm})$ & 11.0 & 13.0 \\
Premoderator thickness $(\mathrm{cm})$ & 1.5 & 1.5 \\
Reflector radius $(\mathrm{cm})$ & 70 & 70 \\
Reflector height $(\mathrm{cm})$ & 70 & 70 \\
Cold neutron $\left(\mathrm{n} / \mathrm{cm}^{2}\right.$ Sr MW s) & $1.74 \times 10^{12}$ & $1.59 \times 10^{12}$ \\
\hline
\end{tabular}

externally cooled by water. A first approach assumes a stationary target, but several of them are placed on a rotary platform, so target substitution can be done very quickly. This concept could be considered as a intermediate step between fully rotatory and stationary options. Its main advantages are the use of a well known technology, with 15 years of operational experience accumulated in SINQ and its compatibility with other water based designs options (e.g., the cold plates design). So far, neutron efficiency has been assumed to be the weak point.

In order to analyze this concept, two independent MCNPX target models have been developed. Fig. 10 shows the model proposed by ESS-Bilbao which considers lead rods with $1 \mathrm{~cm}$ of diameter, $0.75 \mathrm{~mm}$ of zircalloy cladding and a $1 \mathrm{~mm}$ gap between rods. In the side view, the water inlet and outlet channels are visible.

Table 4 shows the optimal design parameters for both models, using water and heavy water as coolants. The results obtained with both models are coherent.

Fig. 11 shows the results in terms of neutron brightness for both models and coolants. The use of heavy water increases brightness values due to the reduction of neutronic absorptions, up to values comparable with the results for the lead-bismuth concept. 


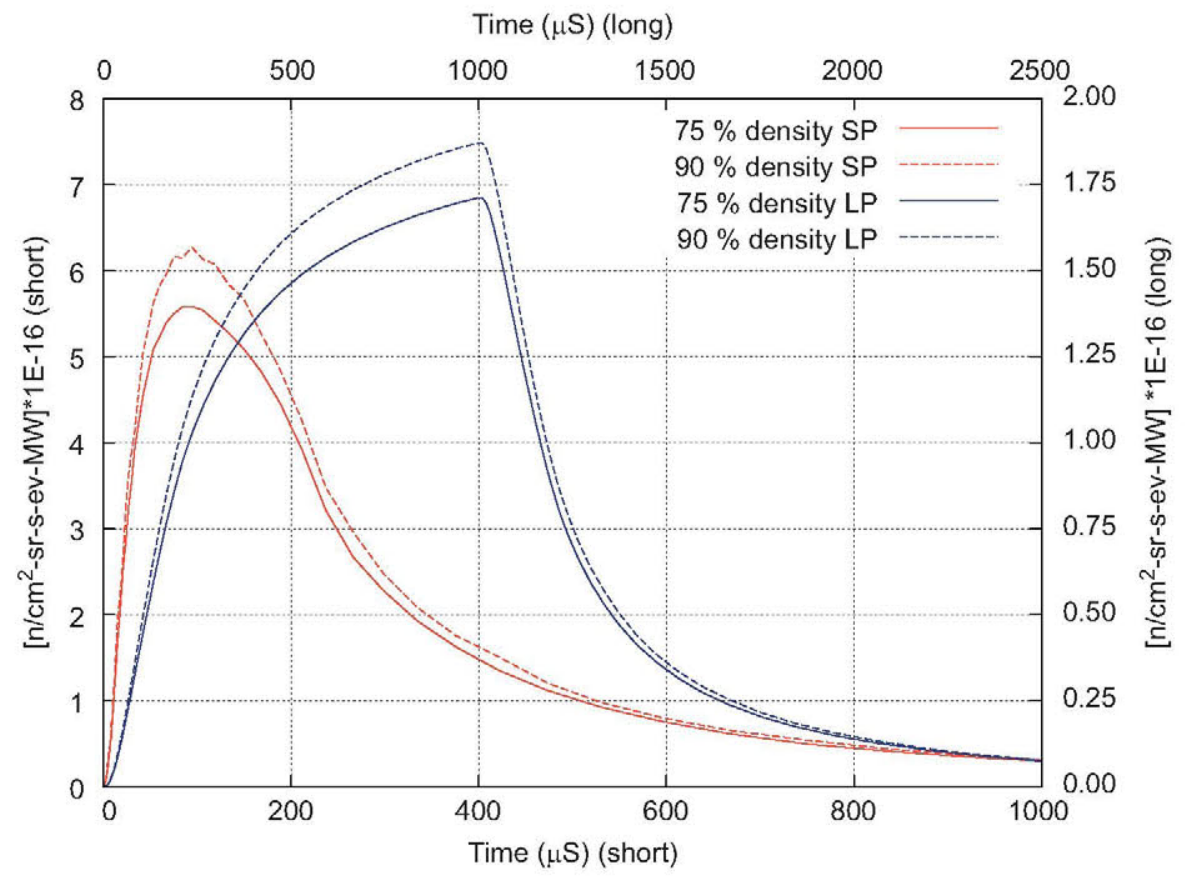

Fig. 9. Brightness for helium cooled solid targets.

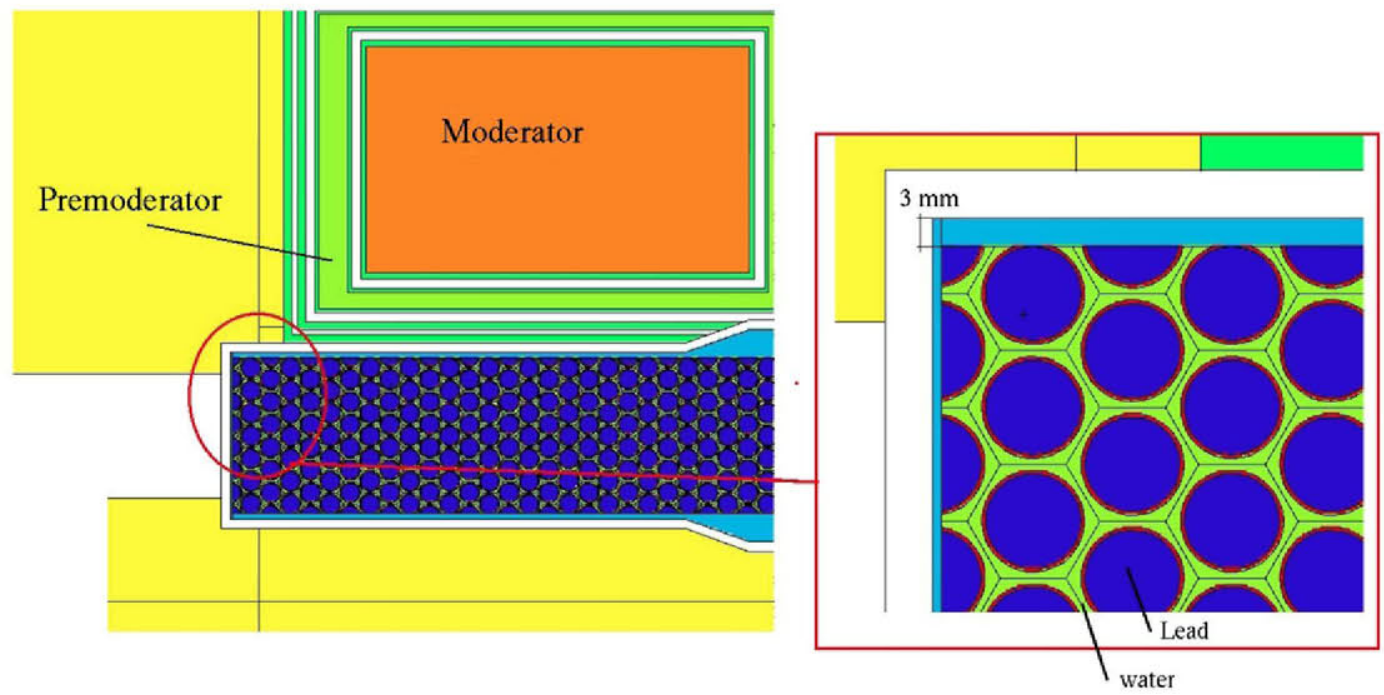

Fig. 10. Cannelloni target geometry.

Table 4

Final parameters after optimization process for cannelloni target.

\begin{tabular}{lll}
\hline Parameter & H2O ESS-B & D20 ESS-B \\
\hline Moderator radius $(\mathrm{cm})$ & 10.0 & 10.0 \\
Relative position $(\mathrm{cm})$ & 17.0 & 16.5 \\
Premoderator thickness $(\mathrm{cm})$ & 0.9 & 0.9 \\
Reflector radius $(\mathrm{cm})$ & 70 & 70 \\
Reflector height $(\mathrm{cm})$ & 70 & 70 \\
Cold neutron $\left(\mathrm{n} / \mathrm{cm}^{2}\right.$ Sr MW s) & $1.31 \times 10^{12}$ & $1.53 \times 10^{12}$ \\
\hline
\end{tabular}

\section{Target comparison}

In the previous sections four target options have been analyzed, and thanks to the optimizations loops, a representative comparison is possible. Fig. 12 shows time distributions of $5 \mathrm{meV}$ neutrons on the moderator surface with $1 \mathrm{~ms}$ pulse for solid and liquid targets.

In order to present a quantitative analysis of the previous figure, the term "signal" is defined as the integral of the time distribution up to $1 \mathrm{~ms}$, and the term "tail", as the integral from $1 \mathrm{~ms}$ to the end of simulation time. Table 5 shows signal and tail for neutrons around $5 \mathrm{meV}$ with relation to a solid rotating target (i.e., taking the signal and tail value of the Solid rotating target as $100 \%$ ). A solid target with cold plates will produce the best neutron performance, with $15 \%$ more signal than lead-bismuth and 25\% less tail distribution. Nevertheless, if target density is reduced, solid rotating targets will produce the same neutronic performance as liquid metals with a lower tail distribution. This result is consistent with the conclusions of other optimization studies [21].

Another possible way to characterize the pulse shape is the time from peak flux to a certain percentage, such as $10 \%$ or $50 \%$. Both times are shown in Table 6 for the targets analyzed. 


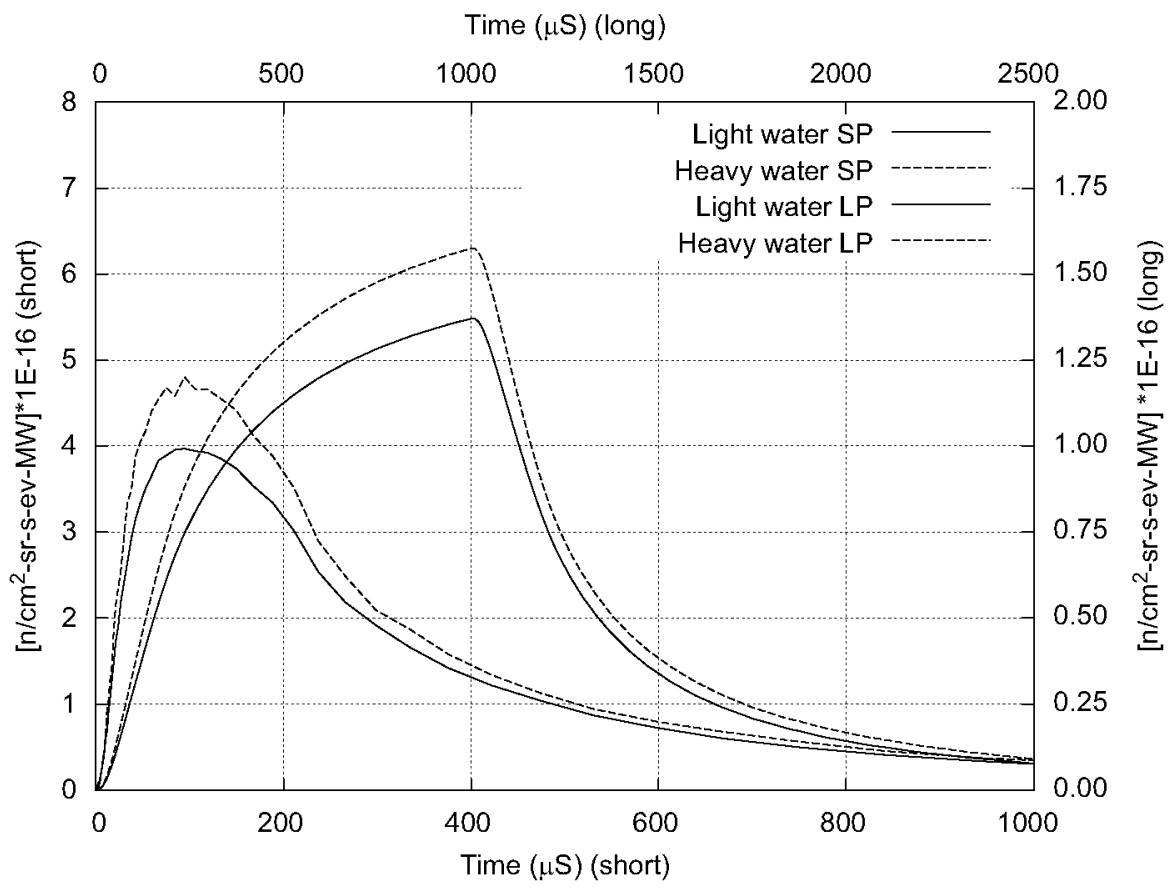

Fig. 11. Brightness for Cannelloni targets.

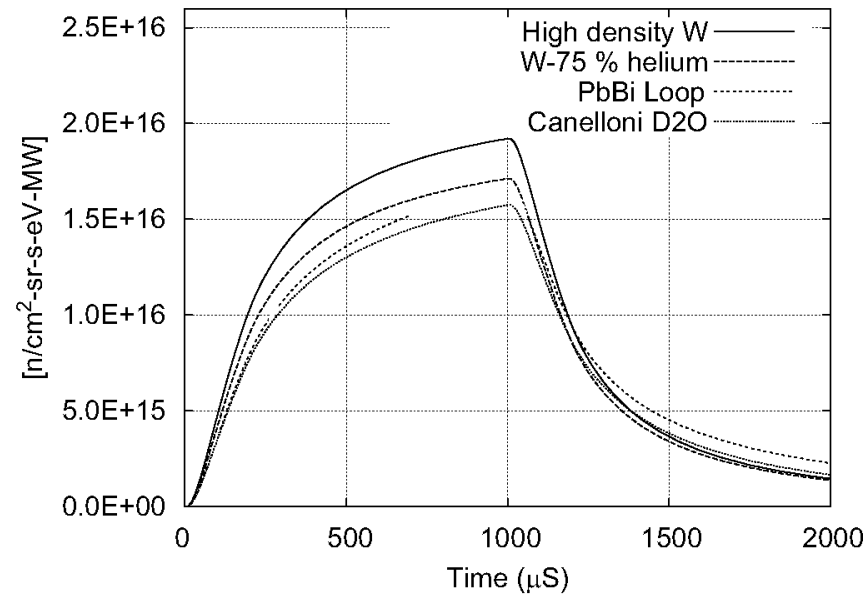

Fig. 12. Brightness comparison for 1 ms length pulse.

Ultimately, it is up to the neutron user to decide which indicator best defines the pulse, given his particular needs, but the qualitative assessment of the pulses is the same: the sharpest pulse with the highest peak is produced with the cold plates target, Helium cooled rotating target produces a flux that is somewhat lower, with a very slightly longer tail, Cannelloni target produces the lowest brightness, with a longer decay time, and liquid metal target produces similar brightness peak than that of Helium cooled, but with a significantly longer tail.

While performance is dependent on many factors, the main reason a cold plates rotating target has the best performance is its high density, which creates a denser source of fast neutrons. This, in turn, allows for a higher cold neutron flux if the moderator is placed correctly. Compared to the other types of targets, we can pinpoint the reasons solid rotating target gives the best performance.

Compared to a Cannelloni target, the reason for the lower brightness in Cannelloni target is the fact that water soaks up part of the proton energy without contributing with neutrons. Cannelloni target does have the advantage of having more reflector around than cold plates, but that is nowhere near enough to make up for the loss of proton energy. If light water is introduced rather than heavy water, it also absorbs some neutrons, further decreasing brightness.

The He-cooled target with $75 \%$ density has lower performance since the density is lower. While no energy is lost to water absorption, the spallation neutrons are more scattered, so the flux in the moderator is lower, resulting in lower brightness. As shown in Table 3, this disadvantage increases as density is reduced, and is further increased if water is added in the target volume, for the same reason that Cannelloni target loses performance. However, if target density can reach $90 \%$ of bulk $\mathrm{W}$, He-cooled target would achieve the same performance as the $95 \%$ density cold plate water cooled options. While the density is slightly lower, there is no loss to water absorption.

The comparison with a liquid metal target is more complex due to the less obvious differences. In order to split the differences, the optimization of an hypothetical tungsten target with the same geometry as the liquid metal targets was performed. Such a target would be impossible to cool, but the results reveal that brightness would be around $12 \%$ higher than the $\mathrm{PbBi}$ target, in other words, we can say that target material gives a $12 \%$ boost to brightness, compared to the lead-bismuth target, thanks to its greater density. Besides, liquid metal target has an extra layer of cladding due to safety issues, that causes cold plates target to have an extra advantage. However, the loss of reflector causes it to drop to around the same integrated flux of mercury. Nevertheless, it is important to notice that, because the neutrons lost come from a part of the reflector, most of them belong to the tail of the distribution, hence why the cold plates have around the same integrated flux, but with a different, sharper time distribution.

\section{Conclusions}

Given the restrictions posed by the current lack of detailed engineering designs, an inherent uncertainty of approximately $10 \%$ for the neutronic yields of all investigated targets has to be 
Table 5

Target neutron performance analysis for $2.5 \mathrm{GeV}$ protons ( $5 \mathrm{meV}$ neutrons).

\begin{tabular}{|c|c|c|c|c|}
\hline Parameter & W Rot & PbBi Loop & $75 \%$ Helium W & Cannelloni D20 \\
\hline Signal (n/cm ${ }^{2}$ eV Sr MW) & $1.4 \times 10^{13}$ & $1.2 \times 10^{13}$ & $1.3 \times 10^{13}$ & $1.1 \times 10^{13}$ \\
\hline Tail (n/cm ${ }^{2}$ eV Sr MW) & $6.5 \times 10^{12}$ & $8.0 \times 10^{12}$ & $6.2 \times 10^{12}$ & $6.6 \times 10^{12}$ \\
\hline Ratio & 2.18 & 1.51 & 2.05 & 1.72 \\
\hline Signal (\% W) & 100 & 85.1 & 90.1 & 80.9 \\
\hline Tail (\% W) & 100 & 123 & 95.6 & 102 \\
\hline
\end{tabular}

Table 6

Peak and decay time characterization for $5 \mathrm{meV}$ neutrons.

\begin{tabular}{|c|c|c|c|c|}
\hline Parameter & W Rot & PbBi loop & 75\% Helium W & Cannelloni D20 \\
\hline Peak value (n/cm $\mathrm{cm}^{2} \mathrm{eV}$ Sr MW s) & $1.89 \times 10^{16}$ & $1.71 \times 10^{16}$ & $1.68 \times 10^{16}$ & $1.37 \times 10^{16}$ \\
\hline Decay time to $50 \%(\mu s)$ & 190 & 230 & 200 & 230 \\
\hline Decay time to $10 \%(\mu s)$ & 810 & 1300 & 860 & 1030 \\
\hline
\end{tabular}

accepted. As basically the obtained neutron yields for the diverse concepts actually only differ in this range it can be concluded that in terms of neutron production possible target types do not differ greatly under ESS conditions. While the advantage of the higher neutron flux must be considered, especially for uses where maximum neutron performance is paramount, the authors do not think it should determine the choice. Other criteria like safety, environmental compatibility, reliability and cost will thus dominate the choice of an ESS target.

As main conclusions of this analysis, it can be stated:

- Solid rotating target cooled by cold plates presents the highest neutron performance for coupled parahydrogen cylindrical moderators.

- Low density (75\% of $\mathrm{W}$ ) helium cooled solid rotating target yields $10-15 \%$ less neutron performance compared to cold plates. At $90 \%$ density, however, the performance would match the cold plates target.

- Lead-bismuth target produces between 15\% and 20\% less useful neutrons on moderator surface than solid rotating cold plate design. It also produces between 20\% and 25\% more neutrons in the tail distribution, so higher background noise should be expected.

- Cannelloni target cooled by heavy water presents similar neutron performance than lead-bismuth target and helium cooled targets, between $15 \%$ and $20 \%$ lower than cold plates concept.

\section{Acknowledgments}

Part of the research leading to these results has received funding from the European Community's Seventh Framework Programme (FP7/2007-2013) under grant agreement No. 202247 "NeutronSourceESS". Extensive and inspiring discussions with many colleagues from a large number of institutions collaborating since the ESS-PP study are gratefully acknowledged. This work has been possible thanks to the support of the computing infrastructure of the i2BASQUE academic network. Fruitful discussions with L. Zanini and M. Wohlmuther (PSI) are gratefully acknowledged. The authors would also like to thank F. Martínez (ESS-Bilbao) for his help in redacting this work, and S. Domingo and F. Gallmeier (SNS) for their MCNPX models.

\section{References}

[1] MCNPX user's Manual. Version 2.6.0, LA-CP-07-1473, Los Alamos National Laboratory, 2007
[2] F.X. Gallmeier, E.B. Iverson, Target Moderator Reflector Assemblies for a SNS Second Target Station, Technical Report, LA-UR-1764, SNS, Oak Ridge, 2009

[3] J. Carpenter, T. Mason, Nuclear Instruments and Methods in Physics Research Section A: Accelerators, Spectrometers, Detectors and Associated Equipment 545 (2005) 1.

[4] Y. Kiyanagi, N. Watanabe, H. Iwasa, Nuclear Instruments and Methods in Physics Research Section A: Accelerators, Spectrometers, Detectors and Associated Equipment 343 (1994) 558.

[5] R. Macfarlane, New Thermal Neutron Scattering Files for ENDF/B-VI Release 2, Technical Report, LA-12639-MS, Los Alamos National Laboratory, 1994.

[6] M. Chadwick, Nuclear Data Sheets 107 (2006) 2931.

[7] R. Forrest, J. Kopecky, J.-C. Sublet, Comprehensive Nuclear Data Library with Covariance Data, JEFF Meeting, OECD/NEA, Paris, France.

[8] S.G. Mashnik, K.K. Gudima, M.I. Baznat, A.J. Sierk, R.E. Prael, N.V. Mokhov, CEM03.S1, CEM03.G1, LAQGSM03.S1, and LAQGSM03.G1 Versions of CEM03.01 and LAQGSM03.01 Event-Generators, Technical Report, LA-UR1764, Los Alamos National Laboratory, March 2006.

[9] M. Harada, N. Watanabe, M. Teshigawara, T. Kai, Y. Ikeda, Nuclear Instruments and Methods in Physics Research Section A: Accelerators, Spectrometers, Detectors and Associated Equipment 539 (2005) 345.

[10] D. Picton, S. Bennington, T. Broome, T. Beynon, Nuclear Instruments and Methods in Physics Research Section A: Accelerators, Spectrometers, Detectors and Associated Equipment 545 (2005) 363.

[11] T. Kai, M. Harada, M. Teshigawara, N. Watanabe, Y. Ikeda, Nuclear Instruments and Methods in Physics Research Section A: Accelerators, Spectrometers, Detectors and Associated Equipment 523 (2004) 398.

[12] B.J. Micklich, E.B. Iverson, Calculation of pulse shapes for reentrant moderators, in: Nuclear Mathematical and Computational Sciences: A Century in Review, A Century Anew, LaGrange Park, Gatlinburg, Tennessee.

[13] E.B. Iverson, Methods for Calculating the Neutronic Performance of Moderators on Pulsed Neutron Sources, 2007, unpublished.

[14] M. Futakawa, K. Haga, T. Wakui, H. Kogawa, T. Naoe, Nuclear Instruments and Methods in Physics Research Section A: Accelerators, Spectrometers, Detectors and Associated Equipment 600 (2009) 18.

[15] T. McManamy, A. Crabtree, D. Lousteau, J. DeVore, L. Jacobs, M. Rennich, Journal of Nuclear Materials 377 (2008) 1. (Proceedings of the Eighth International Workshop on Spallation Materials Technology).

[16] G. Bauer, M. Butzek, H. Conrad, D. Filges, F. Goldenbaum, P. Jung, G. Mank, R. Moormann, The ESS Project, vol. III, Technical Report, ISBN 3-89336-303-3, The ESS Council, 2002.

17] F. Groeschel, L. Cachon, A. Cadiou, T. Dury, C. Fazio, T. Kirchner, G. Laffont, K Thomsen, A. Zucchini, Final Design, Performance Estimation and Safety Analysis of the Megapie Target System, San Diego, CA, United States. Accelerator Driven Systems (ADS); Heat Removal Fluids; Neutron flux; Spallation Neutron Targets, pp. 431-437.

[18] A. Zarraoa-Garmendia, F. Sordo, I. Sard, B. Etxeita, A. Bilbao, G. Bauer, T. McManamy, M. Rennich, Design study of a rotating target for ESS, in: International Collaboration of Advanced Neutron Sources ICANS XIX 19th Meeting, Grindelwald, Switzerland.

[19] T. McManamy, M. Rennich, F. Gallmeier, P. Ferguson, J. Janney, Journal of Nuclear Materials 398 (2010) 35. (Proceedings of the Ninth International Workshop on Spallation Materials Technology).

[20] K. Thomsen, M. Butzek, F. Gallmeier, J. Wolters, Nuclear Instruments and Methods in Physics Research Section A: Accelerators, Spectrometers, Detectors and Associated Equipment 625 (2011) 5.

[21] W. Yin, T.J. Liang, Q.Z. Yu, Nuclear Instruments and Methods in Physics Research A: Accelerators, Spectrometers, Detectors and Associated Equipment 631 (2011) 105 